\title{
Scandium and dimethylaminopyridine catalyzed dehydrative coupling of secondary benzylic and primary alcohols to synthesize unsymmetrical ethers
}

\author{
Julia M. Duncan, ${ }^{1 \dagger}$ Lun Li, ${ }^{1,2+}$ Vahid Mohammadrezaei, ${ }^{1}$ and Laina M. Geary ${ }^{1 *}$
}

\begin{abstract}
We developed a direct catalytic condensation of benzylic alcohols and primary alcohols to synthesize unsymmetrical ethers in one step, catalyzed by scandium triflate and p-dimethylaminopyridine (DMAP). Preliminary experiments give some insight into the mechanism of the reaction, though suggest that the process is quite complex. We suspect the rapid formation of a dimer from a secondary benzylic alcohol via a carbocation intermediate precedes unsymmetrical ether formation.
\end{abstract}

Introduction

The direct modification of functional groups is an ongoing challenge in synthetic organic chemistry. Alcohols are abundant and easily accessible via a variety of transformations and are thus attractive substrates for further modification. However, alcohols typically require derivatization or preactivation to enable their use in coupling or substitution reactions. The desire for chemoselective, direct transformation of alcohols into alternate functional groups has been identified as a key goal in the pharmaceutical industry. ${ }^{1}$ While there are now a number of protocols to do so, ${ }^{2}$ direct condensation of two symmetrical alcohols to form ethers represents a significant challenge. Recent work in the syntheses of unsymmetrical ethers have relied on gold catalysts, ${ }^{3}$ solid-phase thiazolium salts, ${ }^{4}$ ruthenium catalysts, $^{5}$ and boronic acid activation. ${ }^{6}$

We discovered that when 3-hydroxydihydrobenzofuran 1a was exposed to a Lewis acid catalyst and primary alcohol, direct substitution to the unsymmetrical ethers occurred. We report application of this chemistry to a variety of secondary and tertiary benzylic alcohols, as well as some preliminary mechanistic investigations (Scheme 1).

\footnotetext{
${ }^{1}$ University of Nevada, Reno Department of Chemistry; Reno Nevada USA

${ }^{2}$ Westlake University Department of Chemistry; Hangzhou China

† These authors contributed equally to this work
} 
Scheme 1. The synthesis of unsymmetrical ethers via a direct catalytic substitution.

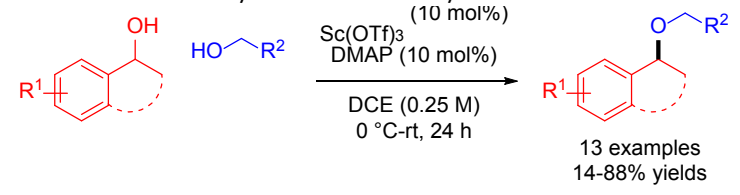

\section{Results and Discussion}

Initial screening studies used 3-hydroxydihydrobenzofuran 1a as substrate for reactions with prenol. Scandium triflate is a well-known Lewis acid catalyst for dehydrogenation of alcohols in a variety of applications. ${ }^{7}$ Appling catalytic amounts of scandium triflate led to full consumption of $1 \mathrm{a}$ at room temperature in acetonitrile after 30 minutes; however, only trace amounts of the desired ether substitution product was detected, along with significant amounts of benzofuran from competing elimination chemistry. Copper, ${ }^{8}$ ytterbium, ${ }^{9}$ and bismuth triflates $^{10}$ all led to incomplete consumption of 1 a under otherwise identical conditions, and yielded only trace amounts of the substitution product, despite precedence for their ability to enable direct ionization of alcohols. Both zinc triflate, able to catalyze the direct cyanation of benzylic alcohols at $100{ }^{\circ} \mathrm{C}^{11}$ and zinc chloride, known for dehydration of alcohols to form ethers at room temperature, ${ }^{12}$ were unreactive towards 1 a at room temperature. Similarly, copper catalysts known to ionize allylic alcohols at room ${ }^{13}$ or elevated temperatures ${ }^{14}$ were ineffective at this dehydrogenative benzylic ether formation from $1 a$.

As the preliminary reactions performed using scandium triflate as the catalyst led to the complete consumption of the 1a, albeit with low selectivity for ether formation, we then screened various reaction conditions in the coupling of 3a with neopentyl alcohol 2a to optimize for formation of the desired ether. Ultimately, we found that performing the reaction in the polar aprotic dichloroethane (DCE) and initiating the reaction at $0{ }^{\circ} \mathrm{C}$ was optimal as increasing the temperature led to complete formation of benzofuran 4, and keeping the reaction at lower temperatures slowed the reaction down substantially. However, in the absence of an exogenous ligand, that was at the expense of formation of ether 3a (Table 1, entry 1). We examined amino acids as ligands, which are an inexpensive source of diverse functionality, with $1 \mathrm{a}$ and neopentyl alcohol $2 \mathrm{a}$ as substrates (Table 1 , entries 2-7). Heteroaromatic L-histidine led to full consumption of $1 \mathrm{a}$ and a modest yield of the desired $3 a$. Applying basic amino acids $L$-lysine and $L$-arginine as ligands improved the relative conversion to $3 a$, though the isolated yield was slightly diminished (entry 3,4$)$. Other aromatic amino acid ligands, L-tryptophan and L-phenylalanine performed poorly, and incomplete consumption of $1 \mathrm{a}$ 
was observed (entry 5,6 ). Although use of glycine as ligand gave comparable product ratios to L-histidine, the yield was poor. We were interested in identifying the particular chemical motif of $L$-histidine that allowed it to function as an effective ligand for selective direct substitution of a benzylic alcohol. Imidazole as a ligand led to poor conversion and selectivity for $3 a$ (entry 8). N-Substitution at the 1 or 3 position of the imidazole ring of L-histidine improved selectivity for $3 a$ and modestly improved yield, but did not give full conversion of 1 a (entries 9,10 ). Converting the carboxylic acid of L-histidine to the methyl ester resulted in an improvement in isolated yield of $3 a$ relative to the parent amino acid (entry 11 vs 2), but the yield was still only moderate at $44 \%$.

Table 1. Selected optimization experiments in the dehydrative coupling of 3-hydroxydihydrobenzofuran 1a to neopentylalcohol $2 \mathrm{a}$. $^{\mathrm{a}}$

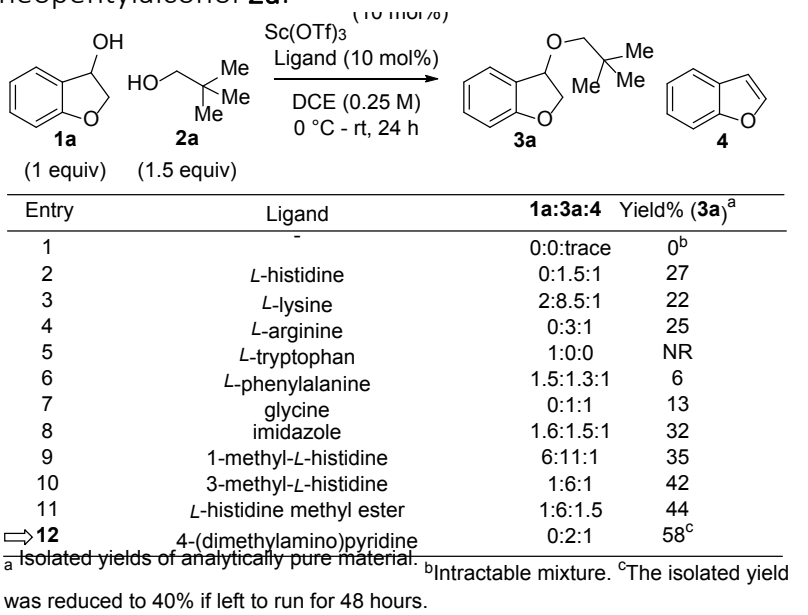

We rationalized that the Lewis basic ligands were modulating the relative Lewis acidity of the scandium catalyst, and were pleased to find that employing 4-dimethylaminopyridine in conjunction with $\mathrm{Sc}(\mathrm{OTf})_{3}$ led to the formation of $3 a$ in 58\% isolated yield (entry 12). Increasing catalyst loading to 20 mol\% did not result in a significant increase in isolated yield of 3a, and decreasing the loading to 5 mol\% only slowed the reaction down, and did not improve selectivity for

\section{3a over 4 .}

The unusual success of DMAP in combination with $\mathrm{Sc}(\mathrm{OTf}) 3^{15}$ promoted us to evaluate other nitrogen bases, both mono- and bidentate (Table 2). Guanidine. $\mathrm{HCl}$ and guanidine derivatives led to exclusive production of benzofuran 4 , unless functionalized with an electron donating group (entries 1-3 versus entries 4-6). Bidentate ligands slowed the consumption of 1a substantially, consistent with decreased reactivity with excess DMAP (vide infra). While the catalyst derived from bipyridines $\mathbf{L} 7$ and $\mathbf{L} 8$ were able to successfully generate some ether $3 a$, the catalysts derived from phenanthrolines L9 and L10 led only to formation of benzofuran 4, perhaps due to the increased basicity of the 
phenanthrolines relative to bypyridines. ${ }^{16}$ Most reactions that employed pyridine-derived ligands produced moderate amounts of ether 3a, with the exception of 2,6-lutidine L16 and 2,6-ditertbutylpyridine L17, which only produced benzofuran $4 .{ }^{17}$ DMAP L13, 2-phenylpyridine L18, and 2,6-bis(diethylamino)pyridine L20 were equally effective, and DMAP L13 was selected for further studies as it is inexpensive, easily handled, and not as susceptible to decomposition as L20. ${ }^{18}$

Table 2. Screening of Lewis basic ligand in conjunction with scandium triflate.

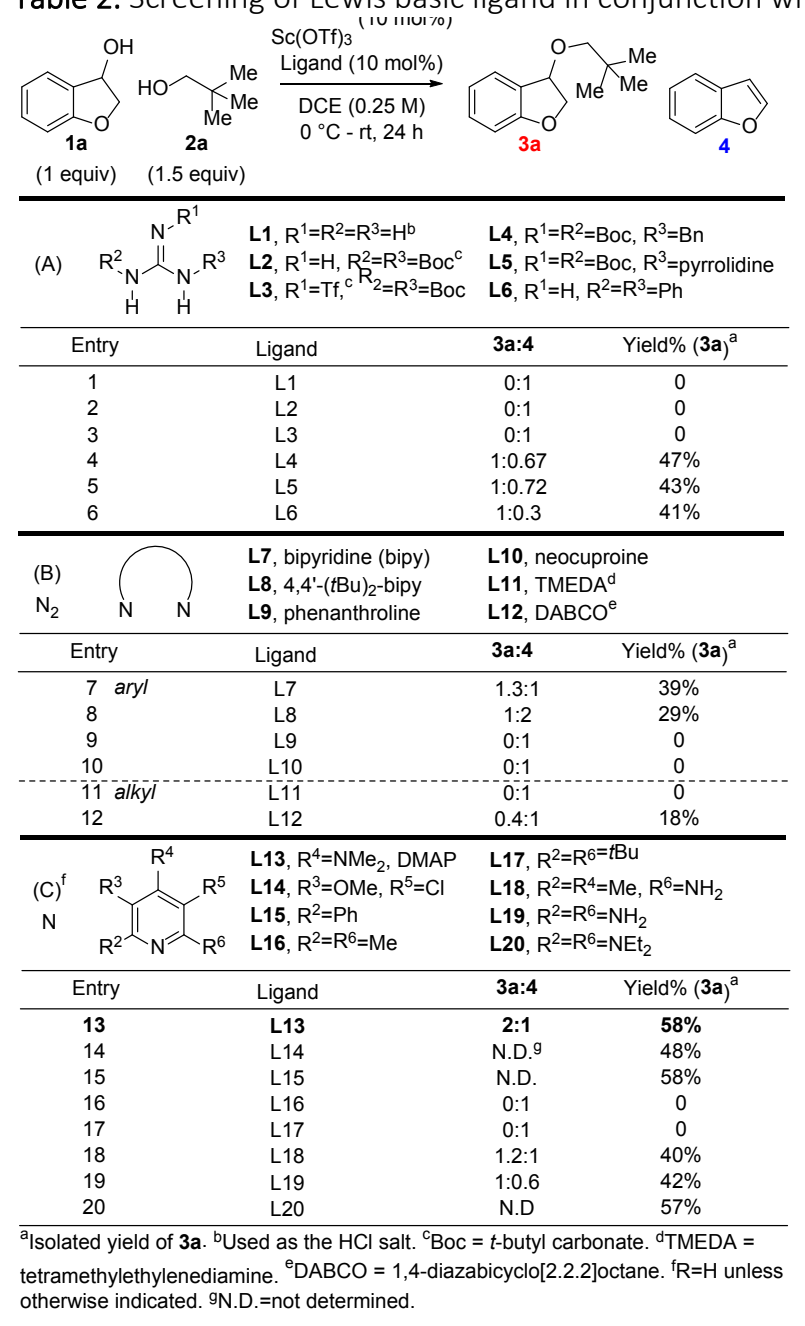

We then finally looked at the effect of DMAP loading relative to scandium (Scheme 2); unsurprisingly, we found that increasing DMAP to 20 mol\% (2 equiv. relative to scandium), the reaction between either 1a or $1 \mathrm{i}$ and neopentyl alcohol $2 \mathrm{a}$ was slowed, and the isolated yields of ethers $3 \mathrm{a}$ and $3 \mathrm{i}$ were reduced. Further increasing the loading of DMAP to 30 mol\% (reaction with 1a) or 50 mol\% (reaction with 1l), the reaction was shut down entirely, and no ether or elimination product could be detected. These results are consistent with the reduced ability of catalysts derived 
from bidentate ligands to promote the reaction, and further supports that the DMAP ligand is tuning the Lewis acidity of scandium to control the reaction and favor substitution reactions.

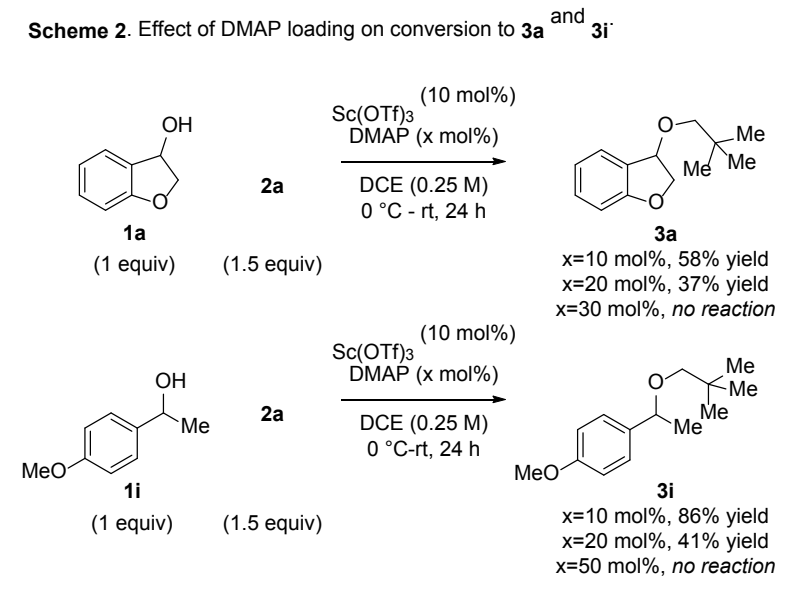

With optimized conditions in hand, we evaluated the scope of the reaction with respect to the nature of the benzylic alcohol component (Table 3). While the reaction between $2 \mathrm{a}$ and $1 \mathrm{a}$ gave ether $3 \mathrm{a}$ in $58 \%$ yield, an analogous 3 hydroxy-5-methoxydihydrobenzofuran 1 b substrate was unreactive under identical conditions. However, 5-bromo-3hydroxydihydrobenzofuran $1 \mathrm{c}$ as substrate gave a modest $37 \%$ yield of the corresponding ether $3 \mathrm{c}$ with incomplete consumption of $\mathbf{1 c}$. One could predict that a carbocation generated from $\mathbf{1 b}$ would be more stabilized and therefore less reactive than a carbocation generated from either $1 \mathrm{a}$ or $1 \mathrm{c} ;^{19}$ given that we have evidence of the possibility of carbocation intermediates (vide infra), that could justify the lack of reactivity of $1 \mathrm{~b}$. The reaction of indanol $1 \mathrm{~d}$ with $2 \mathrm{a}$ gave $50 \%$ yield of ether $3 \mathrm{~d}$ as estimated by the crude ${ }^{1} \mathrm{H}$ NMR spectrum of the reaction mixture, which could not be separated cleanly from the competing elimination product, indene $\mathbf{4 d}$.

We then evaluated the direct substitution of dihydrochromenols and tetrahydronapthalenols in the presence of scandium and DMAP. Dihydrochromenols $1 \mathrm{e}$ and $1 \mathrm{f}$ were suitable substrates, and ethers $3 \mathrm{e}$ and $3 \mathrm{f}$ were formed in $40 \%$ and $70 \%$ yield, respectively, in high conversion and selectivity. However, tetrahydronaphalenol $3 \mathrm{~g}$ was produced in only $26 \%$ yield and reaction with the quaternary $1 \mathrm{~h}$ led gave exclusive elimination to dihydronaphthalene $4 \mathrm{~h}$. 


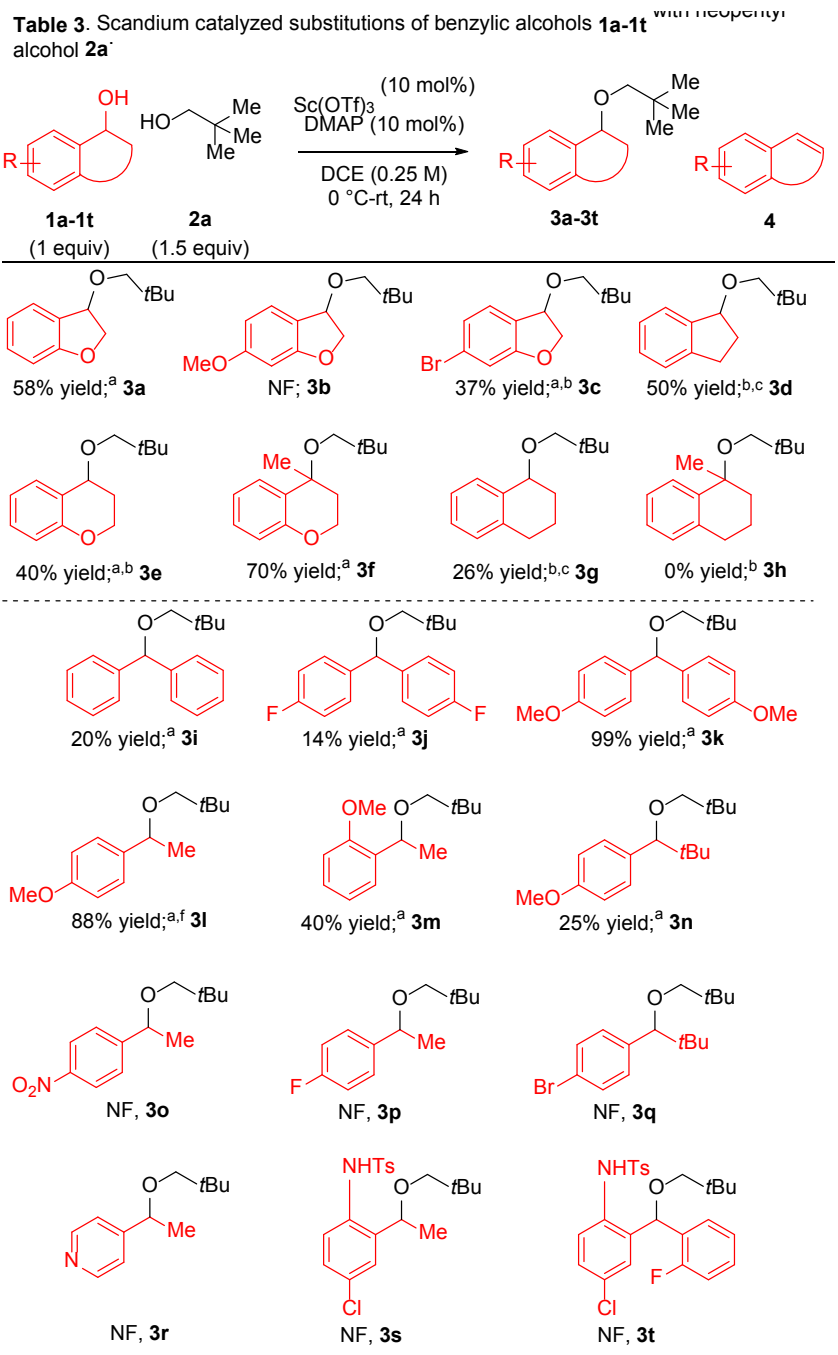

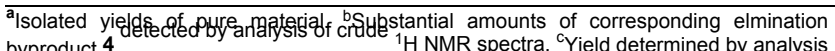
of crude ${ }^{1} \mathrm{H}$ NMR spectra. ${ }^{\mathrm{f}}$ Average of two runs. NF=not formed.

We next evaluated the utility of acyclic benzhydrols and secondary benzylic alcohols in the substitution chemistry. The parent benzyhydrol, as well as the di-p-fluorosubstituted variant were only moderately good substrates, and ethers 3i and $\mathbf{3} \mathbf{j}$ were produced in relatively low yields. In contrast, the bis(p-methoxyphenyl)methanol was an excellent substrate, and ether $\mathbf{3 k}$ was produced in nearly quantitative yield. With those data in hand, we evaluated several secondary benzylic alcohols with one aryl and one alkyl ligand (substrates 1l-1t). Unfortunately, but unsurprisingly, only substrates with a methoxy group were reactive $(1 \mathbf{l}-1 \mathrm{n})$. The reaction was most successful when the methoxy group was in the para position, and ether 31 was isolated in $86 \%$ yield; the more sterically encumbered ethers $3 \mathrm{~m}$ and 3n were isolated in lower yields. Benzylic alcohols with electron withdrawing groups were entirely unreactive under the optimized conditions, consistent with the necessity for generating a cationic intermediate. 
As ether 3 I was produced in high yield, we next examined the scope of the reaction of 1 l with various primary alcohol nucleophiles (Table 4). Most alcohols examined gave moderate to high yields of the unsymmetrical ether (5b-5j). Simply primary and allylic alcohols were effective coupling partners $(2 b-2 d)$ to yield ethers $5 b-5 d$, as were alcohols containing $\beta$-substitution ( $2 \mathrm{e}$ and $2 \mathrm{f}$ to give $5 \mathrm{e}$ and $5 \mathrm{f}$ ). Notably, furfural alcohol $\mathbf{2 g}$ and highly activated benzylic alcohol $2 \mathrm{~h}$ could also be employed, and the unsymmetrical ethers $5 \mathrm{~g}$ and $5 \mathrm{~h}$ were isolated in high yields, with no detection of byproducts arising from ionization of $2 \mathrm{~g}$ or $2 \mathrm{~h}$.

We then sought to employ amino alcohols as coupling partners for direct ether formation. Though unprotected amino alcohols were unreactive in this process, $N$-tosyl-3-aminopropanol $2 \mathrm{i}$ and $N$-tosyl-(S)-valinol $2 \mathrm{j}$ were both able to couple with benzylic alcohol $\mathbf{1} \mathbf{i}$, and ethers $5 \mathbf{i}$ and $\mathbf{5 j}$ were isolated in high yields, albeit with no diastereoselectivity in the case of $5 \mathbf{j}(1: 1 \mathrm{dr})$. An analogous reaction with $N$-Boc valinol was unsuccessful; the consumption of $1 \mathbf{i}$ was incomplete, and the only identifiable products were $p$-methoxystryene and dimer 6.

Table 4. Scandium catalyzed substitution of 4-methoxylphenyl-1-ethanol $1 \mathbf{i}$ with alcohols $2 a-1 i^{a}$
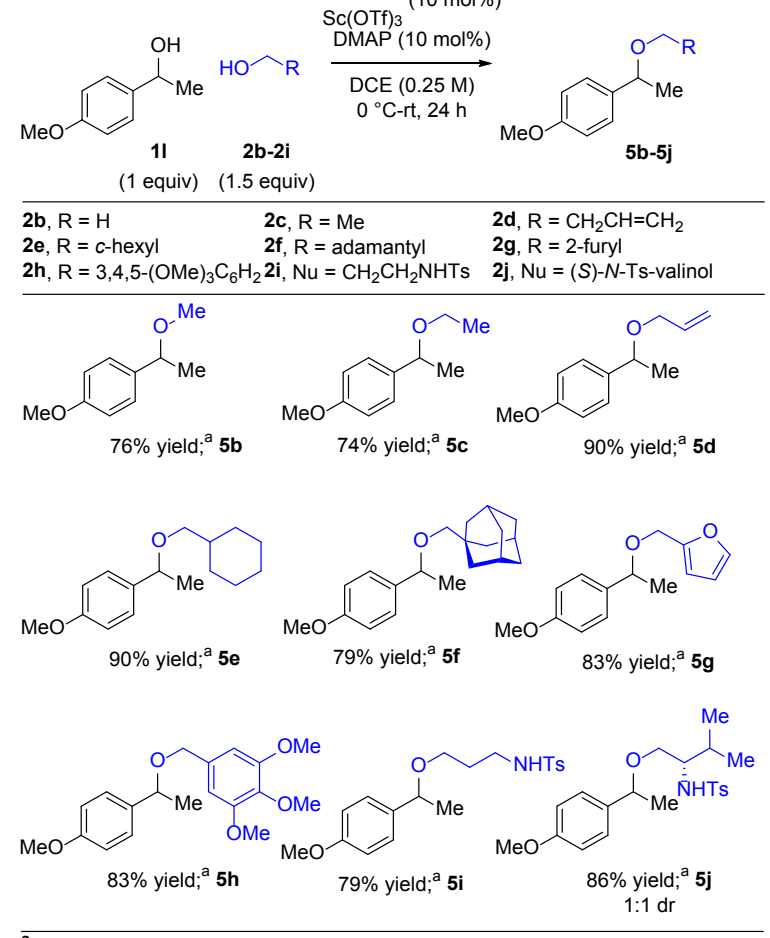

asolated yields of pure material.

In most experiments in Table 4, we couldn't detect any significant amount of elimination product to $p$-methoxystyrene or the product of homodimerization of 11 . However, when the primary alcohol was omitted from the reaction, 
homodimer 6 was isolated in high yields and moderate diasteroselectivity (Scheme 3, equation a). p-Methoxybenzyl alcohol $1 \mathbf{k}$ was unreactive under the standard reaction conditions (equation $\mathrm{b}$ ). Those results suggested that we should have been able to selectively couple an activated benzylic alcohol with an unprotected diol, and thus we evaluated several diol coupling partners with benzylic alcohol 1 l under our optimized conditions (Scheme 3, equations c-e). We found that both 1,2-propane diol (Scheme 3c) and 1,3-butanediol (Scheme 3d) were unreactive under optimized conditions, nor were styrene of dimer 6 detected. We hypothesized that the lack of reactivity was due to the diol chelating the Lewis acid catalyst, rendering it unable to promote any reaction. ${ }^{20}$ We reasoned that a longer chain between the secondary and primary alcohols would be less likely to form a chelate with scandium, and exposed 1i to 1,4-pentanediol under optimized conditions (Scheme 3e). Unlike reactions with primary alcohols that generally went to completion within 20 hours (Table 3), the reaction with 1,4-pentanediol was very slow, and reasonable conversion was observed only after 3 days. We attribute this to some amount of scandium-diol chelate forming; however, this would form a 7-membered chelate, which wasn't as stable as the 5- and 6-membered chelates that would have formed from 1,2-propane and 1,3-butanediols and scandium, respectively. Thus, some active catalyst was present in solution and could promote the reaction between 1 l and pentanediol, yielding 51\% yield of a $2.6: 1$ mixture of $7 \mathrm{a}$ and $7 \mathrm{~b}$ as $1: 1$ mixtures of diastereomers.

To further probe that hypothesis, we employed methoxyethanol as a substrate, and ether 8 was isolated in $43 \%$ yield (Scheme 3f). This supports our hypothesis above, and protecting one of the alcohols makes the deoxygenated substrate a poorer ligand for scandium, enabling the catalytic dehydrogenative coupling. 
Scheme 3. Selectivity for reaction of benzylic alcohol with primary versus secondary alcohols.

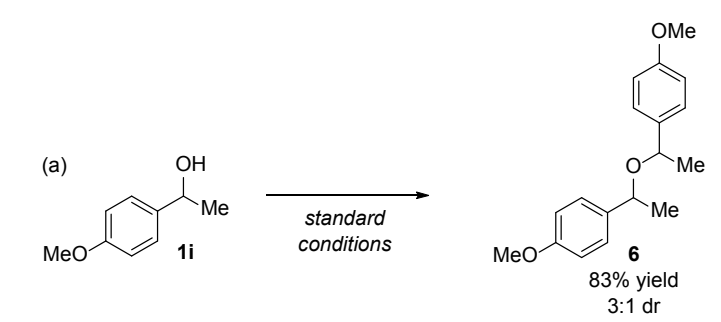

(b)

(c)

$\mathrm{OH}$

$\mathrm{Me} \sim \mathrm{OH}$

$1 \mathrm{i}$

$$
\underset{\text { standard }}{\stackrel{(2 \mathrm{l}, 1.5 \text { equiv) }}{\longrightarrow}}
$$

no reaction

(d)

$$
\overbrace{\mathrm{OH}}^{\mathrm{OH}}
$$

$\stackrel{(2 \mathrm{~m}, 1.5 \text { equiv) }}{\longrightarrow}$

standard

no reaction

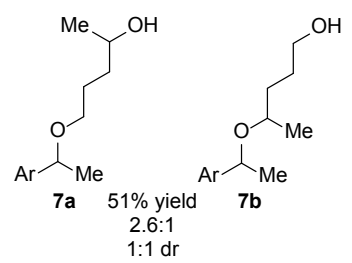

(f)

(e)

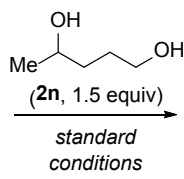

3 days

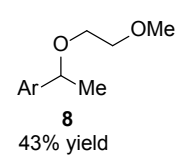

(g)

$\mathrm{OH}$

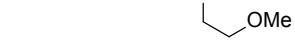

$1 \mathrm{i}$

$$
\underset{\substack{\text { standard } \\ \text { conditions }}}{\stackrel{(21,1.5 \text { equiv })}{\longrightarrow}}
$$

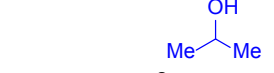

$1 i$

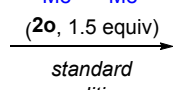
conditions
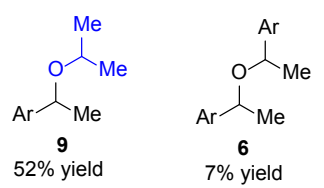

(h)

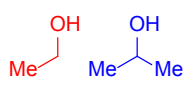

$1 \mathrm{i}$

$$
\underset{\begin{array}{c}
\text { standard } \\
\text { conditions }
\end{array}}{\stackrel{(1.5 \text { equiv each) }}{\longrightarrow}}
$$
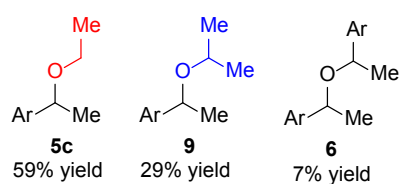
An exogenous secondary aliphatic alcohol was able to selectively react with alcohol 11 , and ether 9 was formed in $52 \%$ yield, with only $7 \%$ of the benzylic dimer 6 isolated (Scheme 3g). This could be due to decreased steric encumbrance about isopropanol relative to benzylic alcohol 1 , increased nucleophilicity of the aliphatic alcohol, or a combination of both factors.

In the intramolecular competition experiment between primary and secondary alcohols, the primary alcohol out competed the secondary alcohol in a 2.6:1 ratio (Scheme 3e); a similar result was observed in an intermolecular competition reaction with alcohol $1 \mathrm{l}$ and an equimolar mixture of ethanol and isopropanol; ether $5 \mathrm{c}$ was favored over ether 9 in a 2:1 ratio, with only a small amount of benzylic dimer isolated (Scheme 3g).

Scheme 4. Control reactions to help elucidate the mechanism

(a)
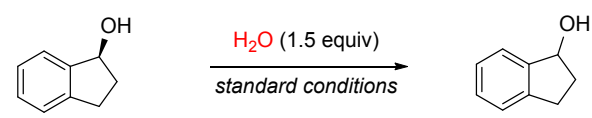

$[\stackrel{(R)-1 d}{=}-17.2$

$\left[\stackrel{( \pm)}{\left.{ }^{\alpha}\right]} \stackrel{-1 d}{=}-0.18\right.$

(1 equiv)

(b)

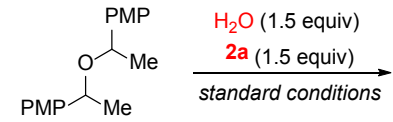

6

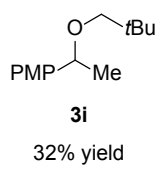

(c)

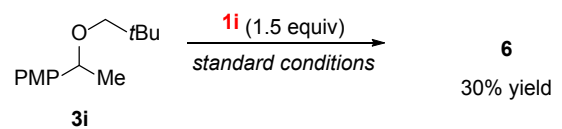

(d)

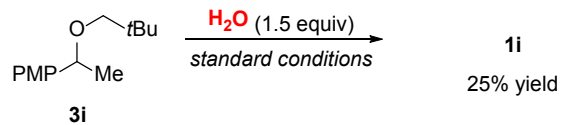

(e)
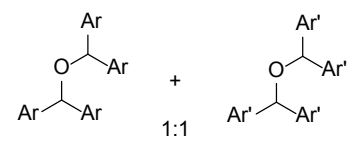

standard no

$10 \mathrm{a}$

$\mathrm{Ar}=4-\mathrm{OMe}-\mathrm{C}_{6} \mathrm{H}_{4}$

10a

$\mathrm{Ar}=4-\mathrm{F}-\mathrm{C}_{6} \mathrm{H}_{4}$

(f)

$\mathrm{PMP} \curvearrowright \underset{\text { standard conditions }}{\stackrel{2 \mathrm{a}(1.5 \text { equiv })}{\longrightarrow}}$

no reaction 
For further mechanistic insight, we subjected enantiopure $(R)$-indanol to the standard reaction conditions in the presence of water as a nucleophile. After approximately 20 hours, the observed optical rotation went from $[\alpha]_{D}^{20}=-$ 17.2 to $[\alpha]_{D}^{20}=-0.18$ (Scheme 4, equation a), suggestive of a carbocation intermediate. We next subjected both the dimer 6 and elimination product to standard reaction conditions to evaluate their role within the catalytic cycle. We found that the dimer 6 was consumed under standard reaction conditions, and was slowly converted into the unsymmetrical ether $\mathbf{3}$ in the presence of neopentyl alcohol $\mathbf{1 l}$ (equation b). Hall ${ }^{21}$ and Gunanathan ${ }^{22}$ recently made similar observations, where the catalytic activation of benzylic alcohols rapidly formed a dimer, which was slowly converted to arylated species via Friedel-Crafts-type reactivity. When ether $\mathbf{3} \mathbf{l}$ was exposed to benzyl alcohol $\mathbf{1} \mathbf{l}$, the dimer 6 could be isolated in $30 \%$ yield (equation c); similarly, when ether $3 \mathbf{I}$ was exposed to water, benzyl alcohol was isolated in $25 \%$ yield (equation d). However, when a mixture of dimers of benzhydrol derivatives were combined in the absence of an alcohol or water nucleophile under otherwise standard reaction conditions, no reaction was observed (equation e), consistent with the necessity of an alcohol or water nucleophile to initiate ionization of the ethers. Similarly, the styrene elimination product was unreactive under the standard reaction conditions (equation $\mathrm{f}$ ).

Thus, we have derived a preliminary mechanistic scenario (Figure 1). The target ether 3 is clearly accessible from dimeric ether 6 , though it is difficult to assess relative rates of formation of 3 from 1 and 6 ( $k_{1}$ vs $\left.k_{3}\right)$ due to the complexity of the reaction. Hydrolysis to starting alcohol 1 occurs from both 3 and 6 ( $k_{-1}$ and k-2, respectively), though those relative rates do not appear to weigh significantly on the overall rate to formation of 3 . The rate of formation of dimer 6 is much faster than direct formation of 3 from $1\left(k_{2}>k_{1}\right)$. Competing elimination appears to only occur from the starting alcohol 1, and formation of the styrene is irreversible and off cycle, as it is not reactive under the reaction conditions. 
Figure 1. Preliminary relative rates of the competing reactions.

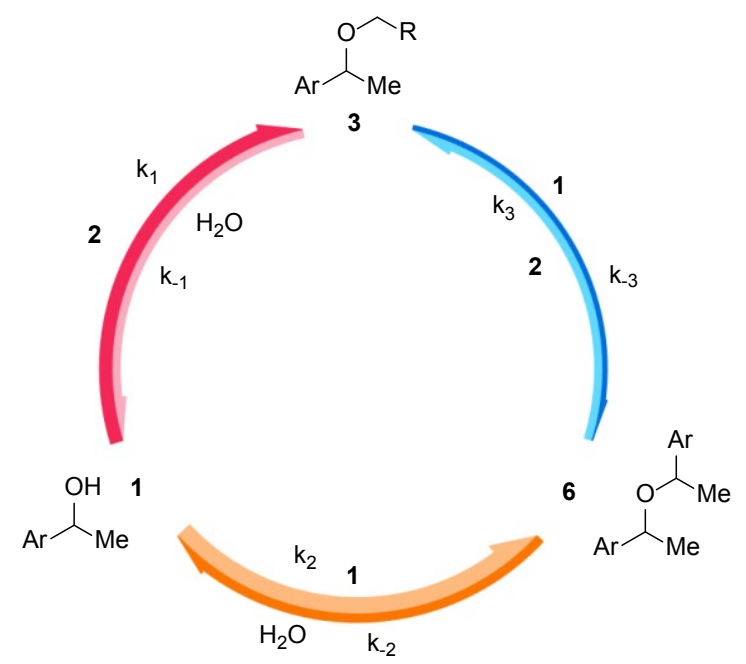

Finally, we were also able to demonstrate that this chemistry may employ aromatic nucleophiles in a Friedel-Crafts type alkylation (Scheme 5). ${ }^{23}$ The reaction between 1a and indole produced $38 \%$ yield of alkylated indole 11. Further examination of aromatic nucleophiles showed promising results towards development of catalytic electrophilic aromatic substitution chemistry, results for which are forthcoming.

Scheme 5. Reaction of indole with a benzylic alcohol via a Friedel-Crafts-like reaction.

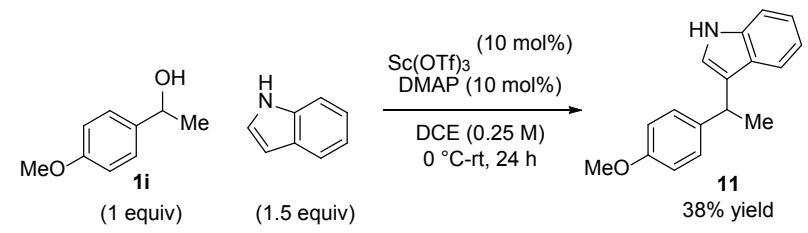

\section{Conclusions}

In summary, we have developed a simple and selective method for producing unsymmetrical ethers via catalytic substitution of benzylic alcohols with primary alcohols. Preliminary mechanistic investigations suggest that the reaction is more $S_{N} 1$-like than $S_{N} 2$-like due to racemization of enantiopure starting alcohols, but the dynamics are complex. The relative rates of formation of dimer 6 and ether 3 are $k_{2}>k_{1}>>k_{3}$, where dimer formation occurs 
faster than unsymmetrical ether from the alcohol 1, and the dimer is much more slowly converted to the ether 3 ;

the overall process has complex kinetics due to competing and reversible side reactions. Further detailed

mechanistic studies are warranted to understand both the nature of the catalyst and the origin of selectivity, and

will be reported in due course.

\section{Acknowledgements}

Acknowledgements The University of Nevada, Reno, and the National Institute of General Medicine of the National

Institutes of Health, Grant R15GM120738 are acknowledged for partial support of this research.

Keywords: scandium $\bullet$ catalysis $\bullet$ dehydration $\bullet$ chemoselective $\bullet$ ethers

\section{References}

1. Constable, D. J. C.; Dunn, P. J.; Hayler, J. D.; Humphrey, G. R.; Leazer, J. J. L.; Linderman, R. J.; Lorenz, K.; Manley, J.; Pearlman, B. A.; Wells, A.; Zaks, A.; Zhang, T. Y. Key Green Chemistry Research Areas-a Perspective from Pharmaceutical Manufacturers. Green Chem. 2007, 9, 411-420.

2. Dryzhakov, M.; Richmond, E.; Moran, J. Recent Advances in Direct Catalytic Dehydrative Substitution of Alcohols. Synthesis 2016, 48, 935-959.

3. Liu, Y.; Wang, X.; Wang, Y.; Du, C.; Shi, H.; Jin, S.; Jiang, C.; Xiao, J.; Cheng, M. Gold(I)-Catalyzed Synthesis of Unsymmetrical Ethers Using Alcohols as Alkylating Reagents. Adv. Synth. Catal. 2015, 357, 1029-1036.

4. Bivona, L. A.; Quertinmont, F.; Beejapur, H. A.; Giacalone, F.; Buaki-Sogo, M.; Gruttadauria, M.; Aprile, C. Thiazolium-Based Catalysts for the Etherification of Benzylic Alcohols under Solvent-Free Conditions. Adv. Synth. Catal. 2015, 357, 800-810.

5. Kim, J.; Lee, D.-H.; Kalutharage, N.; Yi, C. S. Selective Catalytic Synthesis of Unsymmetrical Ethers from the Dehydrative Etherification of Two Different Alcohols. ACS Catal. 2014, 4, 3881-3885.

6. Estopiñá-Durán, S.; Donnelly, L. J.; Mclean, E. B.; Hockin, B. M.; Slawin, A. M. Z.; Taylor, J. E. Aryl Boronic Acid Catalysed Dehydrative Substitution of Benzylic Alcohols for C-O Bond Formation. Chem. Eur. J. 2019, 25, 3950-3956.

7. (a) Matsui, M.; Karibe, N.; Hayashi, K.; Yamamoto, H. Synthesis of A-Tocopherol: Scandium(III) Trifluoromethanesulfonate as an Efficient Catalyst in the Reaction of Hydroquinone with Allylic Alcohol. Bull. Chem. Soc. Jap. 1995, 68, 3569-3571; (b) Tsuchimoto, T.; Tobita, K.; Hiyama, T.; Fukuzawa, S.-i. Scandium(III) Triflate Catalyzed Friedel-Crafts Alkylation with Benzyl and Allyl Alcohols. Synlett 1996, 557-559; (c) El Gihani, M. T.; Heaney, H.; Shuhaibar, K. F. Scandium and Copper Triflate-Catalyzed Acylaminoalkylation and Friedel-Crafts Alkylation Reactions. Synlett 1996, 871-872; (d) Tsuchimoto, T.; Tobita, K.; Hiyama, T.; Fukuzawa, S.-i. Scandium(III) TriflateCatalyzed Friedel-Crafts Alkylation Reactions. J. Org. Chem. 1997, 62, 6997-7005; (e) Noji, M.; Ohno, T.; Fuji, K.; Futaba, N.; Tajima, H.; Ishii, K. Secondary Benzylation Using Benzyl Alcohols Catalyzed by Lanthanoid, Scandium, and Hafnium Triflate. J. Org. Chem. 2003, 68, 9340-9347; (f) Wenz, D. R.; Read de Alaniz, J. Aza-Piancatelli Rearrangement Initiated by Ring Opening of Donor-Acceptor Cyclopropanes. Org. Lett. 2013, 15, 3250-3253.

8. Yadav, J. S.; Reddy, B. V. S.; Rao, T. S.; Rao, K. V. R. Copper(II)-Catalyzed Allylation of Propargylic and Allylic Alcohols by Allylsilanes: A Facile Synthesis of 1,5-Enynes. Tetrahedron Lett. 2008, 49, 614-618.

9. Sharma, G. V. M.; Mahalingam, A. K. A Facile Conversion of Alcohols into P-Methoxybenzyl Ethers (PmbEthers) Using P-Methoxybenzyl Alcohol-Yb(Otf)3. J. Org. Chem. 1999, 64, 8943-8944.

10. (a) Qin, H.; Yamagiwa, N.; Matsunaga, S.; Shibasaki, M. Bismuth-Catalyzed Direct Substitution of the Hydroxy Group in Alcohols with Sulfonamides, Carbamates, and Carboxamides. Angew. Chem., Int. Ed. 2007, 46, 409-413; (b) Zhan, Z.-P.; Yang, W.-Z.; Yang, R.-F.; Yu, J.-L.; Li, J.-P.; Liu, H.-J. BiCl 3 -Catalyzed Propargylic Substitution Reaction of Propargylic Alcohols with C-, O-, S- and N-Centered Nucleophiles. Chem. Commun. 2006, 3352-3354; (c) Rubenbauer, 


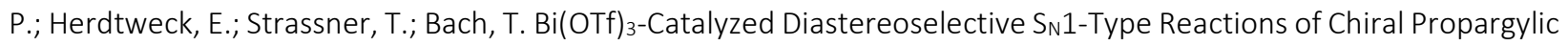
Acetates. Angew. Chem., Int. Ed. 2008, 47, 10106-10109.

11. Theerthagiri, P.; Lalitha, A. Zn(OTf) 2 -Catalyzed Direct Cyanation of Benzylic Alcohols-a Novel Synthesis of A-Aryl Nitriles. Tetrahedron Lett. 2012, 53, 5535-5538.

12. Kim, S.; Chung, K. N.; Yang, S. Direct Synthesis of Ethers Via Zinc Chloride-Mediated Etherification of Alcohols in Dichloroethane. J. Org. Chem. 1987, 52, 3917-3919.

13. Rokade, B. V.; Gadde, K.; Prabhu, K. R. Copper-Catalyzed Direct Transformation of Secondary Allylic and Benzylic Alcohols into Azides and Amides: An Efficient Utility of Azide as a Nitrogen Source. Eur. J. Org. Chem. 2015, 2015, 2706-2717.

14. Chen, K.; Chen, H. J.; Wong, J.; Yang, J.; Pullarkat, S. A. Copper(li) Triflate Catalyzed Allylic Arylation of Allylic Alcohols: Direct and Selective Access to C-Allylanilines. ChemCatChem 2013, 5, 3882-3888.

15. DMAP and Scandium are most often used in in acylation reactions; for an example, see: Greenwald, R. B.; Pendri, A.; Zhao, H. Tetrahedron: Aysmm. 1998, 9, 915-918

16. Kulkari, B., S.; Mishra, D.; Pal, S. Role of Substituents on the Reactivity and Electron Density Profile of Diimine Ligands: A Density Functional Theory Based Study. J. Chem. Sci. 2013, 125, 1247-1258.

17. This could be a steric effect; if the bulkier ligands render the catalyst unable to pi-pi stack, elimination could out compete reaction with an alcohol to form the ether.

18. Both L19 and L20 must be recrystallized immediately before use.

19. Mayr, H.; Bug, T.; Gotta, M. F.; Hering, N.; Irrgang, B.; Janker, B.; Kempf, B.; Loos, R.; Ofial, A. R.; Remennikov, G.; Schimmel, H. Reference Scales for the Characterization of Cationic Electrophiles and Neutral Nucleophiles. J. Am. Chem. Soc. 2001, 123, 9500-9512.

20. It is also possible that the diols were forming intramolecular hydrogen bonds, rendering them unreactive under standard conditions.

21. Ang, H. T.; Rygus, J. P. G.; Hall, D. G. Two-Component Boronic Acid Catalysis for Increased Reactivity in Challenging Friedel-Crafts Alkylations with Deactivated Benzylic Alcohols. Org. Biomol. Chem. 2019, 17, 6007-6014.

22. Sahoo, P. K.; Gawali, S. S.; Gunanathan, C. Iron-Catalyzed Selective Etherification and Transetherification Reactions Using Alcohols. ACS Omega 2018, 3, 124-136.

23. (a) McCubbin, J. A.; Krokhin, O. V. Organocatalyzed Friedel-Crafts Arylation of Benzylic Alcohols. Tetrahedron Letters 2010, 51, 2447-2449; (b) McCubbin, J. A.; Hosseini, H.; Krokhin, O. V. Boronic Acid Catalyzed Friedel-Crafts Reactions of Allylic Alcohols with Electron-Rich Arenes and Heteroarenes. J. Org. Chem. 2010; (c) Wilcke, D.; Herdtweck, E.; Bach, T. Enantioselective Brønsted Acid Catalysis in the Friedel-Crafts Reaction of Indoles with Secondary Ortho-Hydroxybenzylic Alcohols. Synlett 2011, 2011, 1235-1238; (d) Wang, W.; Xiong, W.; Wang, J.; Wang, Q.-A.; Yang, W. Brønsted Acid-Catalyzed Asymmetric Friedel-Crafts Alkylation of Indoles with Benzothiazole-Bearing Trifluoromethyl Ketone Hydrates. J. Org. Chem. 2020, 85, 4398-4407. 